\title{
SIGNIFICANT LIFETIME AND BACKGROUND IMPROVEMENTS IN PEP-II BY REDUCING THE 3RD ORDER CHROMATICITY IN LER WITH ORBIT BUMPS*
}

\author{
F.-J. Decker, Y. Nosochkov, M. Sullivan, G. Yocky, SLAC, U.S.A.
}

\section{Abstract}

Orbit bumps in sextupoles are routinely used for tuning the luminosity in the PEP-II B-Factory. Anti-symmetric bumps at a pair of identical sextupoles separated by $-\mathrm{I}$ section generate the net dispersion, while symmetric horizontal bumps induce a tune shift and beta beat. By combining two of these symmetric bumps with opposite signs, where the second pair is $90^{\circ}$ away, the tune shift cancels and the beta beat doubles. In the low energy ring (LER), there are four -I sextupole pairs per arc, located one after another $90^{\circ}$ apart, where pairs 1 and 3 are at the same phase and pairs 2 and 4 are $90^{\circ}$ away. By making two symmetric bumps with opposite sign in pairs 1 and 3 , the tune shift and beta beat outside this region cancel, but there is a local change of phase and beta in the $2^{\text {nd }}$ sextupole pair located in the middle. By using this bump knob, the LER lifetime improved by a factor of 3, losses by a factor of 5 , and the beam-beam background in the drift chamber of the BaBar detector by $20 \%$. Optics analysis showed that the local phase change at the $2^{\text {nd }}$ sextupole pair can compensate the $3^{\text {rd }}$ order chromaticity.

\section{INTRODUCTION}

Compensation of linear optics errors using feed-down from sextupole orbit bumps has been successfully used in PEP-II [1]. Recently, we have found that the sextupole feed-down can also create a large non-linear chromatic effect helping to compensate the $3^{\text {rd }}$ order chromaticity in LER, which was not thought of in the design phase.

\section{LINEAR EFFECTS OF TUNING BUMPS}

Closed bumps in sextupoles were already used in the SLC final focus tuning [2] and Fig. 1 is a quick reminder of the effects. In the LER, four of the six arcs contain 4 sextupole pairs each, two with focusing sextupoles (SFs) and two with SDs, each one containing two sextupole families. So the arc with SDs looks like the following with the SD1s having half the strength of the SD2s:

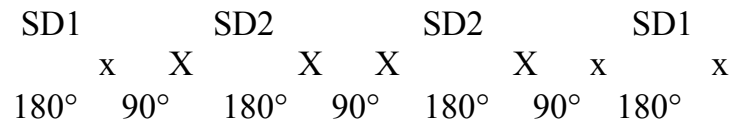

An x-bump in a sextupole will create a feed-down normal quadrupole, while a y-bump makes a skew quadrupole. Combining two of these bumps in a symmetric or an anti-symmetric fashion changes the lattice parameters (see Tab. 1). A single anti-symmetric orbit bump changes dispersion. The strongest bump for $\mathrm{x}$ dispersion would be an anti-symmetric x-bump in the

\footnotetext{
* Work supported by Department of Energy contract DE-AC0276SF00515.
}

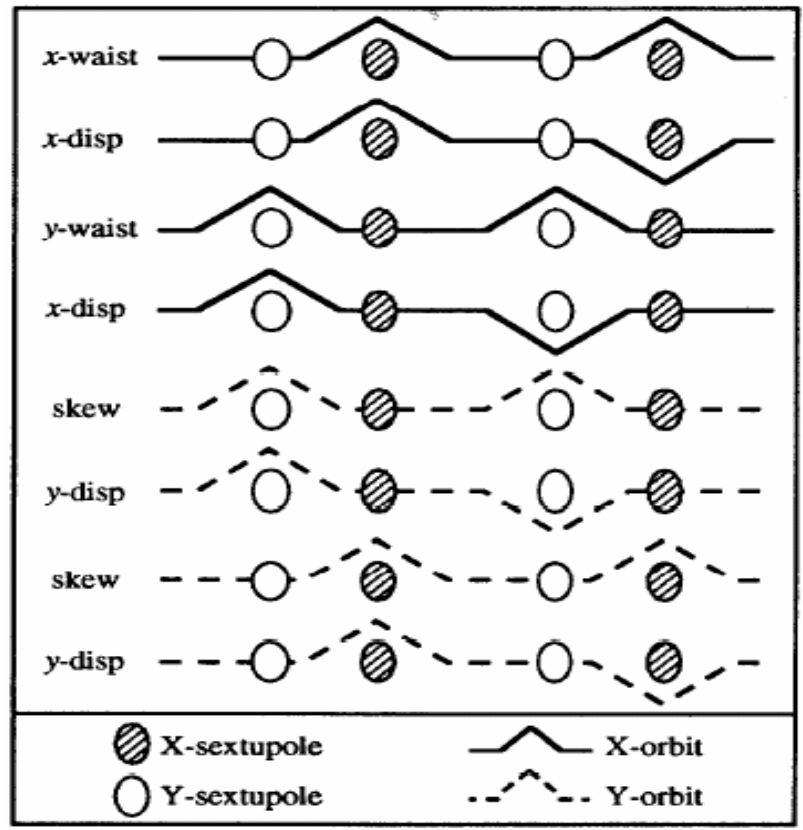

Figure 1: Graphical effects of sextupole bumps.

Table 1: Sextupole bumps change lattice functions

\begin{tabular}{|c|l|l|}
\hline & \multicolumn{1}{|c|}{ SF } & \multicolumn{1}{c|}{ SD } \\
\hline anti-sym $x$ & x-dispersion & x-dispersion (weak) \\
\hline sym x & x-beta beat / phase & y-beta beat / phase \\
\hline anti-sym y & y-dispersion (weak) & y-dispersion \\
\hline sym y & x-y coupling & x-y coupling \\
\hline
\end{tabular}

SF2s, while the strongest bump for y-dispersion is an antisymmetric y-bump in the SD2s. A single symmetric bump affects the beam ellipses. For $\mathrm{x}$ bumps it is beta beat and phase shift. In SF2s the effect is mainly for the x-plane, while in SD2s it is mainly for the y-plane. For y-bumps it is $x-y$ coupling, both SD2s and SF2s are required to control the four coupling parameters. Figure 2 shows the sensitivity, where a symmetric bump in y at the SD2s of about $50 \mu \mathrm{m}$ reduces the luminosity by $2.5 \%$.

Combining more of these basic bumps in y can create bumps which for instance can change the four coupling terms at the interaction point. The beta beat and phase advance shift can be adjusted separately. For example, by combining two symmetric $\mathrm{x}$ bumps anti-symmetrically $(++--)$, a beta beat is created, while the change of phase advance is canceled.

So far the bumps at SD1s or SF1s were not very emphasized since these sextupoles are twice as weak and would require a bigger orbit bump to get the same effect as in SD2s and SF2s. 


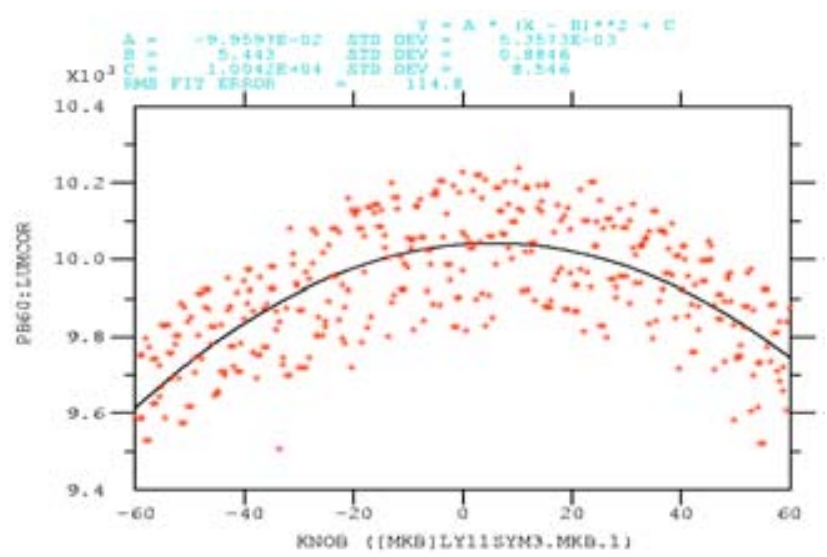

Figure 2: Sensitivity of luminosity to tuning bumps.

\section{NON-LINEAR EFFECTS OF BUMPS}

By design, the four - I sextupole pairs in each LER arc self-cancel the $1^{\text {st }}$ order chromatic beta beat. The ratio of 2:1 for the SF2:SF1 and SD2:SD1 sextupole strengths was selected to further minimize the $2^{\text {nd }}$ order chromatic aberrations. More sextupole families would enable us to cancel more chromatic terms.

\section{Idea of Lifetime Bumps}

Since we don't have more sextupole families which can be individually adjusted, we thought we can change the beta function at some of the sextupole pairs to effectively change their strength. This can be done by making symmetric x-bumps at the SD2 and SD1 of the same phase, with the ratio of $-1 / 2: 1$, e.g.: $0 \quad 0 \quad-1 / 2-1 / 200011$.
Such a bump knob would cancel the global beta beat and tune shift, but would change beta and phase at the sextupoles in between ( $3^{\text {rd }}$ pair in this case).

\section{Experimental Implementation}

Trying these bumps on the running machine with the BaBar detector turned on was an instant success. Not only were the losses at the septum reduced and the LER lifetime improved, but also less background was generated in the detector.

Figure 3 shows the losses at the vertical septum when dialing in the first $-400 \mu \mathrm{m}$ and $+200 \mu \mathrm{m}$ bump combination (of course in the wrong direction initially).

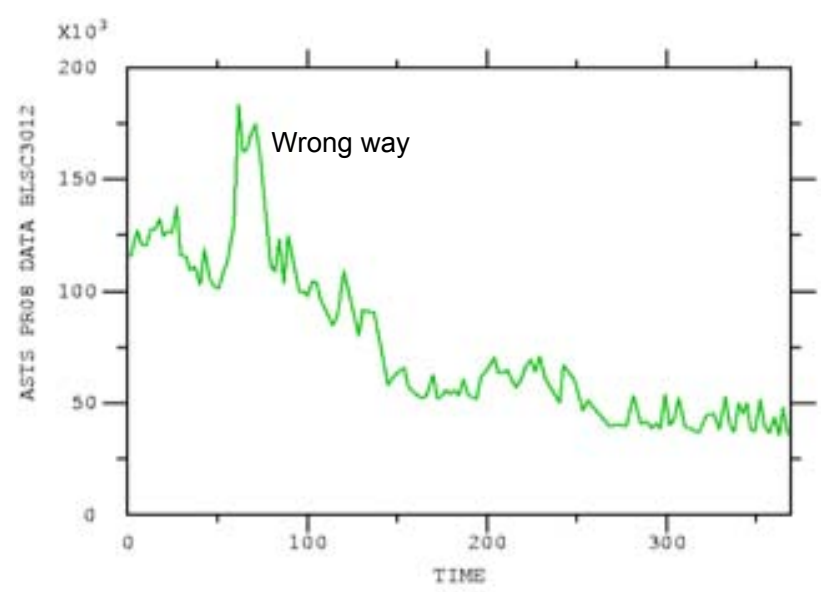

Figure 3: Beam losses at septum versus time in seconds during adjustments of the first bumps.



Figure 4: Reduced drift chamber background (white): $-12 \%$ and $-8 \%$ for two bumps. 
Altogether three of these bump combinations in different arcs with SDs were used to minimize the needed orbit excursion. Figure 4 shows the drift chamber $(\mathrm{DCH})$ background during this implementation. The final results showed a factor of 5 reduction in losses, a factor of 3 in lifetime, and $20 \%$ less BaBar drift chamber background:

$\begin{array}{lrlr}\text { Less loss at septum: } & 120,000 & \rightarrow 20,000 & \text { [counts] } \\ \text { More lifetime: } & 45 & \rightarrow 150 & \text { [min] } \\ \text { Less DCH background: } & 1000 & \rightarrow & 800 \text { [counts] }\end{array}$

Since then we seldom see the low LER lifetimes of 2040 min anymore and the drift chamber background stayed low. Also the y-tune could be lowered to get more luminosity without facing a bad trade-off of too low of a lifetime. Similar bump combinations were used in the SFs arcs but their effects were not as dramatic.

\section{THIRD ORDER CHROMATICITY}

The effect of the SD1, SD2 bump knob on non-linear chromaticity was investigated using a model in Fig. 5, where the SD2(D4) and SD1(D4) pairs (in red) have the symmetric bumps with ratio of $-1: 2$. The only significant linear optics effects are the local change of beta and phase at the SD2(D3) pair in the middle. By studying equivalent models in MAD which allowed independent change of beta and phase at SD2(D3), it was found that the local beta beat had a small effect, but the local phase shift was very effective in correction of the $3^{\text {rd }}$ order $y$ chromaticity. Figure 5 shows that phase at SD2(D3) changes with respect to other sextupoles and therefore voids the design cancellation of $1^{\text {st }}$ order chromatic beta, and gives rise to the $3^{\text {rd }}$ order chromatic tune shift. Table 2 compares the $1^{\text {st }}, 2^{\text {nd }}$ and $3^{\text {rd }}$ order chromaticity in the design lattice, in the model with independent beta and phase changes, and in the actual knob, called D4. It is striking that the sign of the 3rd order can be reversed by this knob and that the empirically found amount more or less brings this value to zero.

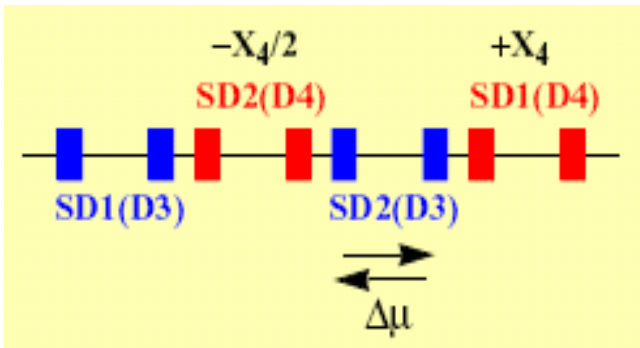

Figure 5: Local phase shift created by D4 knob.

Table 2: Chromaticity change in the model and knob

\begin{tabular}{|l|c|c|c|c|}
\hline & ideal lattice & ideal $+\Delta \beta_{\mathrm{y}}$ & ideal $+\Delta \mu_{\mathrm{y}}$ & $\mathrm{D} 4 \mathrm{knob}$ \\
\hline$\xi_{1 \mathrm{y}}$ & 0.007 & 0.050 & 0.009 & 0.047 \\
\hline$\xi_{2 \mathrm{y}}$ & 176.0 & 173.5 & 160.5 & 159.5 \\
\hline$\xi_{3 \mathrm{y}}$ & 5667 & 5833 & -1333 & -83 \\
\hline
\end{tabular}

Figure 6 shows the tune versus energy offset for the design lattice, while Fig. 7 shows the much flatter $3^{\text {rd }}$ order dependence of the y-tune after the sextupole bump combination is turned on.

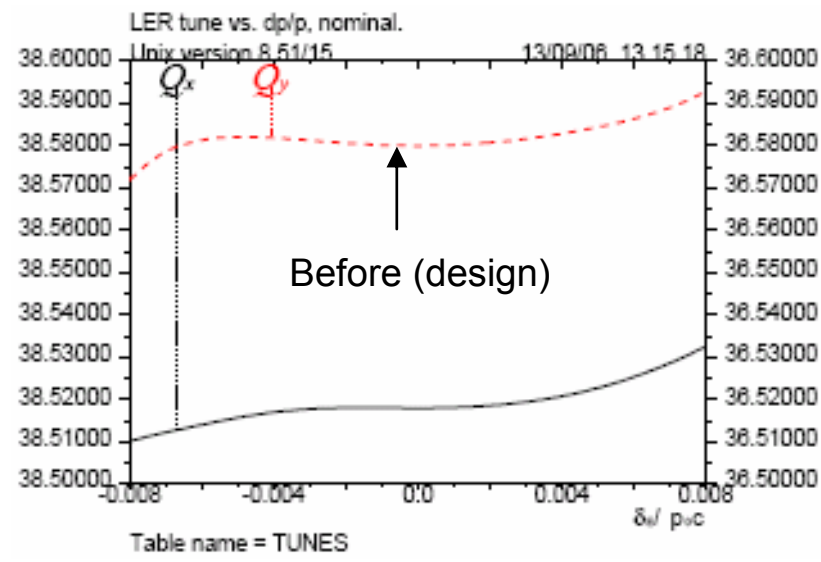

Figure 6: Tune curves for the design LER lattice.

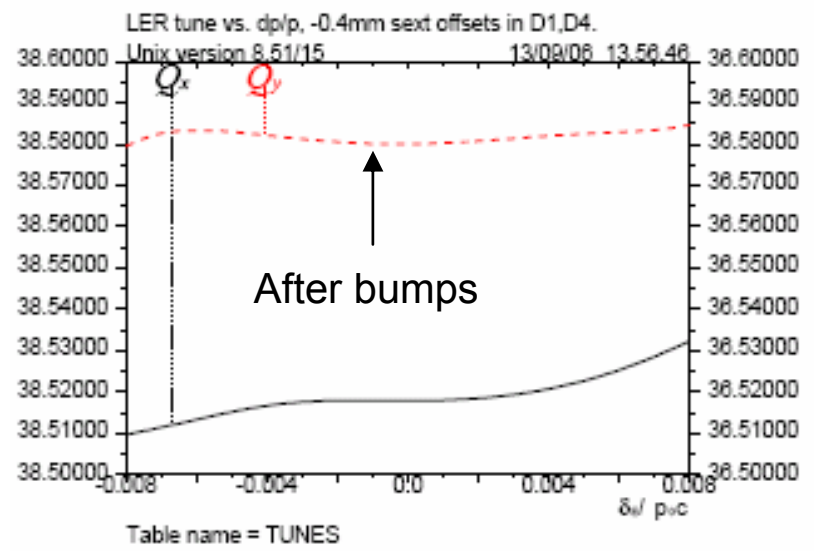

Figure 7: Tune curves after the bumps, showing reduced $3^{\text {rd }}$ order y-tune chromaticity.

\section{SUMMARY}

Combing two symmetric $\mathrm{x}$-bumps in sextupole pairs in a way which locally cancels their first order effects, such as beta beat and phase shift, affected the beam tails so that the beam lifetime in LER increased by a factor of three and detector background improved by $20 \%$. Optics analysis showed that the reason for the improvement is the lower $3^{\text {rd }}$ order chromaticity achieved mainly by the change of betatron phase at the sextupole pair located between the two sextupole bumps.

\section{REFERENCES}

[1] F.-J. Decker et al., "Orbit Distortions and Bumps in the PEP-II LER Ring," EPAC'02, Paris, June 2002.

[2] P. Emma et al., "Beam-based Alignment of the SLC Final Focus Sextupoles", SLAC-Pub-6209, May 1993. 